\title{
Structural basis for differential recognition of brassinolide by its receptors
}

\author{
Ji She ${ }^{1,2}$, Zhifu Han ${ }^{3}$, Bin Zhou ${ }^{4}$, Jijie Chai ${ }^{3,5} \bowtie$ \\ ${ }^{1}$ School of Life Sciences, Peking University, Beijing 100871, China \\ ${ }^{2}$ National Institute of Biological Sciences, Beijing 102206, China \\ ${ }^{3}$ School of Life Sciences, Tsinghua University, Beijing 100084, China \\ ${ }^{4}$ College of Biological Sciences, China Agricultural University, Beijing 100083, China \\ ${ }^{5}$ Tsinghua-Peking Center for Life Sciences, Beijing 100084, China \\ $\triangle$ Correspondence: chaijj@mail.tsinghua.edu.cn \\ Received April 2, 2013 Accepted April 22, 2013
}

\begin{abstract}
Brassinosteroids, a group of plant steroid hormones, regulate many aspects of plant growth and development. We and other have previously solved the crystal structures of BRI1(LRR) in complex with brassinolide, the most active brassinosteroid identified thus far. Although these studies provide a structural basis for the recognition of brassinolide by its receptor BRI1, it still remains poorly understood how the hormone differentiates among its conserved receptors. Here we present the crystal structure of the BRI1 homolog BRL1 in complex with brassinolide. The structure shows that subtle differences around the brassinolide binding site can generate a striking effect on its recognition by the BRI1 family of receptors. Structural comparison of BRL1 and BRI1 in their brassinolide-bound forms reveals the molecular basis for differential binding of brassinolide to its different receptors, which can be used for more efficient design of plant growth regulators for agricultural practice. On the basis of our structural studies and others' data, we also suggest possible mechanisms for the activation of BRI1 family receptors.
\end{abstract}

KEYWORDS X-ray crystallography, leucine-rich repeat domain, brassinosteroid, BRL1

\section{INTRODUCTION}

Steroid hormones are employed both by animals and plants. Brassinosteroids, a group of plant steroid hormones, are essential for growth and development of plants (Clouse and Sasse, 1998). While steroid hormones are perceived by in- tracellular receptors in animals, brassinosteroid-insensitive 1 (BRI1), a membrane protein, has been established as the major receptor for brassinosteroids in plants (Li and Chory, 1997; He et al., 2000; Wang et al., 2001; Kinoshita et al., 2005). BRI1 belongs to a family of leucine-rich repeat receptor like kinases (LRR-RLKs) with more than 200 members in Arabidopsis (Shiu and Bleecker, 2001). A typical structure of proteins in this family contains an extracelluar LRR domain, a single-pass transmembrane region and an intracelluar kinase domain. While many LRR-RLKs have unknown functions, BRI1 and its signaling pathway are well characterized (Vert et al., 2005; Kim and Wang, 2010; Wang et al., 2012). Upon brassinosteroid binding, BRI1 kinase is activated to a basal level, which results in phosphorylation and release of BRI1 kinase inhibitor 1 (BKI1) from the membrane (Wang and Chory, 2006; Jaillais et al., 2011b). BRI1-associated kinase 1 (BAK1), another LRR-RLK with only five LRRs, thus interacts with and fully activates BRI1 ( $\mathrm{Li}$ et al., 2002; Nam and Li, 2002; Wang et al., 2008). Then BRI1 triggers a signaling pathway and regulates the expression of downstream genes. Nevertheless, it is still not fully clear how $\mathrm{BRI} 1$ is activated upon ligand binding.

Brassinosteroids are critical for vascular development (Fukuda, 2004). Consistently, the bri1 mutants in Arabidopsis and rice both have abnormal vascular phenotype (CanoDelgado et al., 2004; Nakamura et al., 2006). All the three homologs of BRI1 in Arabidopsis (BRL1, BRL2 and BRL3) are specifically expressed in vascular cells (Clay and Nelson, 2002; Cano-Delgado et al., 2004), albeit with some different patterns. BRL1 and BRL3 have been suggested to promote xylem differentiation and repress phloem formation (CanoDelgado et al., 2004), while loss of VH1/BRL2 causes defective vein pattern formation and abnormal vascular transport in 
leaves (Clay and Nelson, 2002; Ceserani et al., 2009). BRL1 and BRL3, but not BRL2, rescue the phenotype of bri1 mutants when overexpressed (Cano-Delgado et al., 2004; Zhou et al., 2004). Supporting the functional studies, biochemical assays showed that BRL1, BRL3 and BRI1 strongly bind to brassinolide with dissociation constants of $3.6 \pm 0.07 \mathrm{nmol} / \mathrm{L}, 53.4 \pm$ $0.04 \mathrm{nmol} / \mathrm{L}$ and $55 \pm 0.08 \mathrm{nmol} / \mathrm{L}$ (Cano-Delgado et al., 2004), respectively. In contrast, BRL2 showed no specific brassinolide binding activity (Cano-Delgado et al., 2004).

Structural studies of free BRI1(LRR) and its complex with brassinolide provide important insight into steroid hormone recognition by plants (Hothorn et al., 2011; She et al., 2011). BRI1 harbors an LRR domain interrupted by an island domain. Brassinolide induces and binds to a hydrophobic pocket between the non-LRR island domain and the inner surface of several neighboring C-terminal LRRs of BRI1. However, the mechanism underlying the differential binding of brassinolide to its receptors remains less well understood. Here we report the crystal structure of BRL1(LRR) in complex with brassinolide and a modeled structure of the brassinolide binding domain of BRL2. The structure reveals the molecular basis for the different binding affinities of the BRI1 family of receptors with brassinolide. Based on the structural studies and others' data, we also suggest possible mechanisms for the activation of BRI1 family receptors.

\section{RESULTS}

\section{Overall structure of BRL1(LRR)}

The ectodmain of the BRL1(LRR) (residues 25-758) was expressed and purified as previously described (She et al., 2011). The structure of BRL1(LRR) (residues 31-60, 64-757) in complex with brassinolide was solved to $2.5 \AA$ Asing molecular replacement (Table 1). Structure superposition showed that the structure of BRL1(LRR) is similar to that of BRI1(LRR) (Hothorn et al., 2011; She et al., 2011) (Fig. 1). Like BRI1(LRR), BRL1(LRR) was monomeric in solution (data not shown) and structure. BRL1(LRR) contains 24 LRRs and forms a righthanded superhelix with a similar size to BRI1(LRR) (Fig. 1B). While the convex side of BRL1 consists of helices or loops, the concave side is composed of 24 parallel $\beta$ strands of regular LRRs and one antiparallel $\beta$ strand from $\mathrm{N}$-terminal cap (Fig. 1A). As observed in the BRI1(LRR) structure (Hothorn et al., 2011; She et al., 2011) and another plant LRR protein PGIP2 (Di Matteo et al., 2003), the $\beta$ strands on the convex side also exist in BRL1(LRR) (Fig. 1A). This is likely due to the plant-specific consensus sequence L/fXGxl/vP (X and $x$ stand for polar and any amino acids respectively) (Fig. 2) found in many LRR-RLKs. The $\mathrm{N}$ - and C-terminal caps, like those of BRI1(LRR), shield the two hydrophobic ends of the LRR solenoid from solvent (Fig. 1A). Seven potential glycosylation sites (Asn97, Asn157, Asn227, Asn257, Asn362, Asn532 and Asn558) are defined by sufficient electron density (Figs. 1B and 2). Four of them are conserved in BRI1(LRR) at the structural level (Fig. 1B). Interestingly, the carbohydrate moieties of
Table 1. Summary of diffraction data and structure refinement statistics

\begin{tabular}{|c|c|}
\hline \multicolumn{2}{|l|}{ Data collection statistics } \\
\hline Beam line & Beam line BL17U1, SSRF \\
\hline Space group & C 121 \\
\hline Wavelength ( $\AA$ ) & 1.0 \\
\hline Number of reflections & $75248(3753)^{a}$ \\
\hline Cell dimensions & $\begin{array}{l}a=105.86 \AA \hat{A}, b=83.40 \AA \hat{,}, c=264.06 \AA \hat{~} \\
\alpha=90.00^{\circ}, \beta=97.24^{\circ}, \gamma=90.00^{\circ}\end{array}$ \\
\hline Resolution $(\AA)$ & $2.50(2.54-2.50)$ \\
\hline Rsym (\%) & $9.2(38.8)$ \\
\hline $\mathrm{I} / \sigma(\mathrm{I})$ & $11.75(4.22)$ \\
\hline Completeness (\%) & $94.8(96.6)$ \\
\hline Redundancy & 2.8 \\
\hline \multicolumn{2}{|l|}{ Refinement statistics } \\
\hline Resolution $(\AA)$ & $29.8-2.50(2.53-2.50)$ \\
\hline No. reflections & $74841(2602)$ \\
\hline $\mathrm{R}_{\text {work }} / \mathrm{R}_{\text {free }} \%$ & $17.8 / 23.4(24.47 / 34.56)$ \\
\hline All protein atoms & 11850 \\
\hline \multicolumn{2}{|l|}{ R.m.s deviations } \\
\hline Bond lengths $(\AA)$ & 0.008 \\
\hline Bond angles $\left({ }^{\circ}\right)$ & 1.503 \\
\hline \multicolumn{2}{|l|}{ Ramachandran (\%) } \\
\hline Preferred region & 95.35 \\
\hline Allowed region & 4.65 \\
\hline
\end{tabular}

${ }^{a}$ Highest resolution shell is shown in parenthesis.

both Asn532 and Asn558 in BRL1(LRR) but not Asn532 itself as observed in BRI1(LRR) pack against the island domain (Fig. 1B) and appear to have a role in stabilizing the conformation of the brassinolide binding site. Seven of the eight disulfide bonds are conserved between BRL1(LRR) and BRI1(LRR), except Cys471-Cys498 present in BRL1(LRR) (She et al., 2011) (Figs. 1A and 2). Like that of BRI1, the island domain of BRL1 locates in concave side and comprises three antiparallel $\beta$ strands and a $3_{10}$-helix (Fig. 3A).

\section{Brassinolide recognition by BRL1}

Brassinolide well defined by electron density (Fig. 3B and 3D) binds to a hydrophobic pocket formed between the island domain and the inner surface of the solenoid of BRL1(LRR), which highly resembles that of BRI1(LRR) with an RMSD of $0.76 \AA$ over aligned $205 \mathrm{CA}$ around the binding pocket (Fig. 3A). Brassinolide, in particular the distal side chain, adopts a similar extended conformation when binding to BRL1(LRR) and BRI1(LRR) (Fig. 3C). Most of the interactions, including hydrophobic interactions and hydrogen bonding, of brassinolide with BRI1(LRR) and BRL1(LRR) are highly conserved (Hothorn et al., 2011; She et al., 2011) (Figs. 3C and 4). 

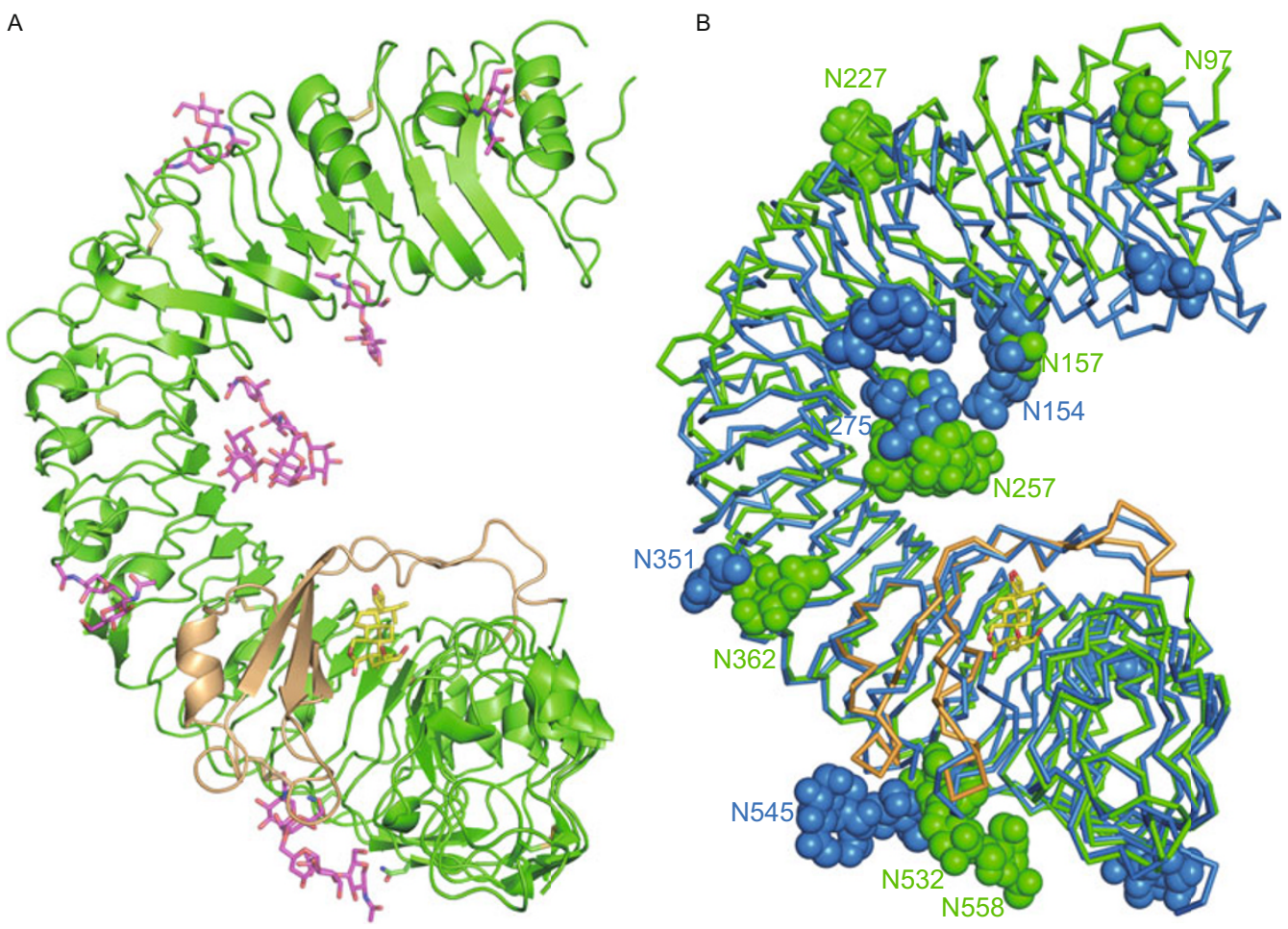

Figure 1. Overall structure of BRL1(LRR). (A) LRRs of BRL1 are shown in green and cartoon. The island domain is colored in orange and the brassinolide molecule in yellow. The N-linked sugars and disulphide bonds are shown in magenta and orange sticks, respectively. (B) Structural alignment of BRI1(LRR) (residues 538-770, marine blue ribbon) and BRL1(LRR) (residues 525-755, green ribbon). Spheres indicate the N-linked sugar groups in BRI1 (marine blue) and BRL1 (green).

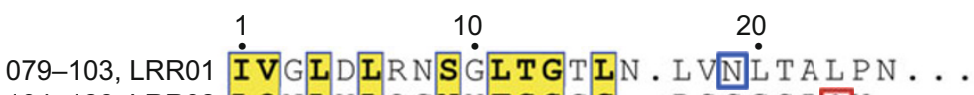
104-126, LRR02 LQN L Y L Q G Y F S S G G . D S S G S DCY . . . . 127-152, LRR03 LQVLDLS S N S I S D Y SMV D Y V F S KCS N . . . 153-176, LRR04 LVSVNI S NNKLVGKL. . G F A P S S L. . Q S . 177-202, LRR05 L T TVDL S Y N I L S D K I. . P E S F I S D F P A S . 203-227, LRR06 L K Y L D L T H N L S G DF . . S D L S F G IC]GN. . 228-252, LRR07 L T F $\mathbf{F}$ S L S Q N N L S G D K F . P I T L P N C K F . . . 253-278, LRR08 LETINI S RNNLAGKI. . P NGE YWGS F Q . 279-303, LRR09 L K L S L A H NR L S GE I . . P P E L S L LCKK T . . 304-327, LRR10 L V I L D L S GN T F S GE L . . P S Q F T ACV W . . . 328-352, LRR11 LQN LN L GNNYLSGDF. . L T TVV K I T G . . 353-376, LRR12 I T Y L Y VA Y N N I S G SV. . P I S L T N C S N . . . 377-403, LRR13 LRV L D L S N G F T GNV. . P S G EC]S L Q S S P V 404-427, LRR14 L E K I L I A N N Y L S G T V.. P ME L G KCK S . . . 428-451, LRR15 L K T I D L F N E L T G P I. . P K E I WM L P N . . . 452-476, LRR16 L S L V MWANN L T G T I. . P E G VCVVKG GN . 477-500, LRR17 LET L I L N N L LT T S I. . P E S I S RCT N . . . 501-524, LRR18 MIWI S L S S NRI T GKI. . P S G I GNLSK . . . 525-548, LRR19 LA I L L GNNS L S GNV . . PRQ L GN C K S . . . 549-572, LRR20 L I W L D I S N N L T G D L. . P GE L A S Q A G . . . 641-664, LRR21 M I Y F D I S Y N Av S G F I. . P P G Y GNMG Y . . . 665-688, LRR22 LQV LN L G HNRITGTI. . P D S F G G L KA. . . 689-712, LRR23 I GV L D L S HNN L Q GY L . . P G S L G S L S F . . . 713-736, LRR24 LS L LVS NNNL T GPI. . P F G G L T T F . . .
\end{abstract}

Figure 2. Sequence alignment of LRRs in BRL1. The boundary of each LRR is shown on the left. The conserved residues are shown with yellow background. Red and blue squares highlight cysteines forming disulfide bonds and glycosylated asparagines respectively. Black solid squares indicate the amino acids specific to plant LRR proteins. 
A
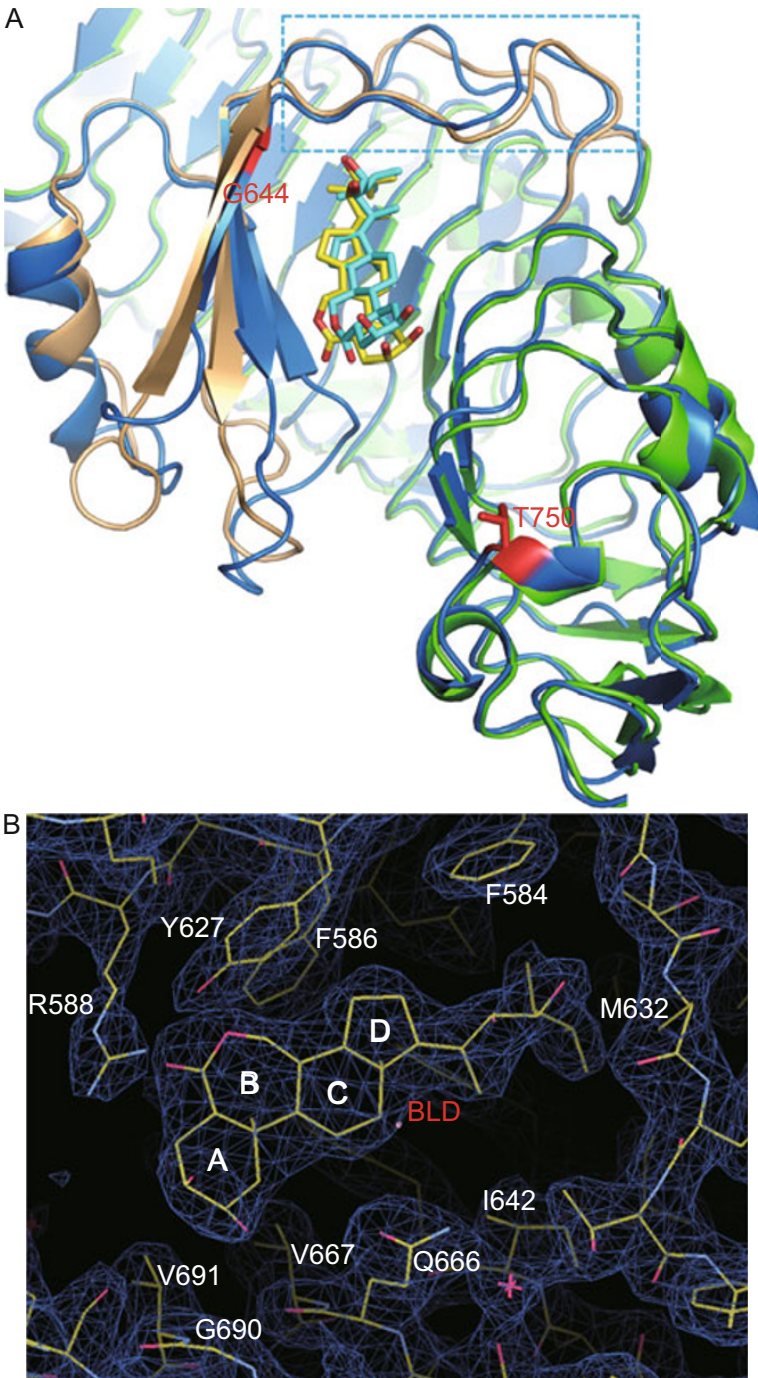

C

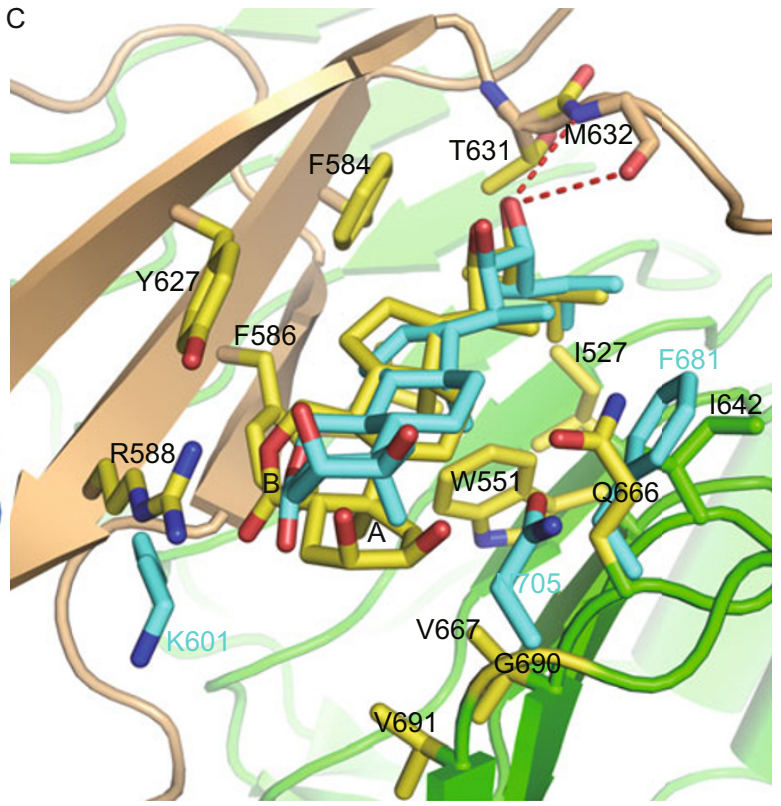

D

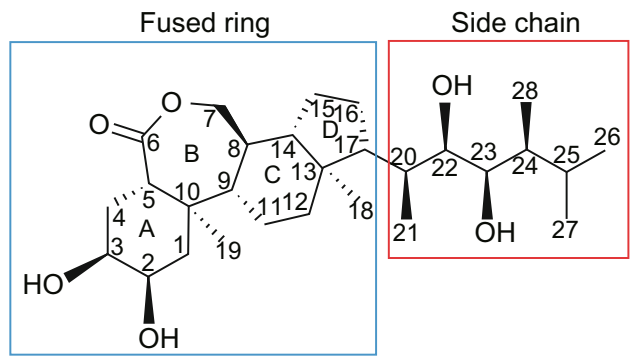

Figure 3. Differential brassinolide recognition by BRL1(LRR) and BRI1(LRR). (A) Structural alignment of brassinolide binding pockets of BRL1 and BRI1. The island domain of BRL1 is colored in orange and LRRs of BRL1 in green. BRI1 is shown as marine blue cartoon. Brassinolides bound to BRL1 and BRI1 are depicted as yellow and cyan sticks, respectively. Blue dashed square highlights the loops connecting island domain and LRRs. Residues of two BRI1 mutants are labeled in red. (B) Electron density map around brassinolide (BLD) binding site. (C) Shown are the structural differences between the brassinolide binding pockets of BRI1(LRR) and BRL1(LRR). Brassinolide and residues in BRI1 are depicted in cyan. The island domain of BRL1 is colored in light orange. Brassinolide and side chains of residues comprising the hormone binding pocket of BRL1 are shown in yellow sticks. Red dashed lines represent hydrogen bonds. (D) Chemical structure of brassinolide. The carbon atoms are labeled as indicated.

As in the BRI1(LRR), the fused ring moiety of brassinolide (ring $A-D)$ occupies most of the binding surface in the structure of BRL1(LRR) (Fig. 3C). Phe586 and Tyr627 of BRL1(LRR) interact with the island domain side of the fused ring moiety (Fig. 3C), while Gln666 in BRL1(LRR), substituting Phe681 in BRI1, establishes Van Der Waals contacts with the LRRs side of brassinolide (Fig. 3C). Nearly perpendicular to the fused ring moiety, the distal side chain (C24-28) of brassinolide anchors to a hydrophobic cavity formed by lle527 and Trp551 from LRRs side and Phe584, Leu601 and Thr631 from island domain side (Fig. 5A). As in BRI1(LRR), the C23 hydroxyl group forms hydrogen bonds, but with the backbone nitrogen and oxygen of Met632 in BRL1(LRR) (Fig. 3C).

Notable structural differences, however, occur to the end of the fused ring portion (Fig. $3 \mathrm{C}$ ). Compared to that in $B R I 1(L R R)$, this region of brassinolide binds deeper into the conserved pocket of BRL1(LRR), leading brassinolide to bury a larger surface area in BRL1(LRR) (388 $\left.\AA^{2}\right)$ than in BRI1(LRR) (360 $\left.\AA^{2}\right)$. These observations suggest a higher binding affinity of brassinolide with BRL1 than with BRI1, supporting previous biochemical data (Cano-Delgado et al., 2004). While most of the brassinolide-interacting residues from the is- 

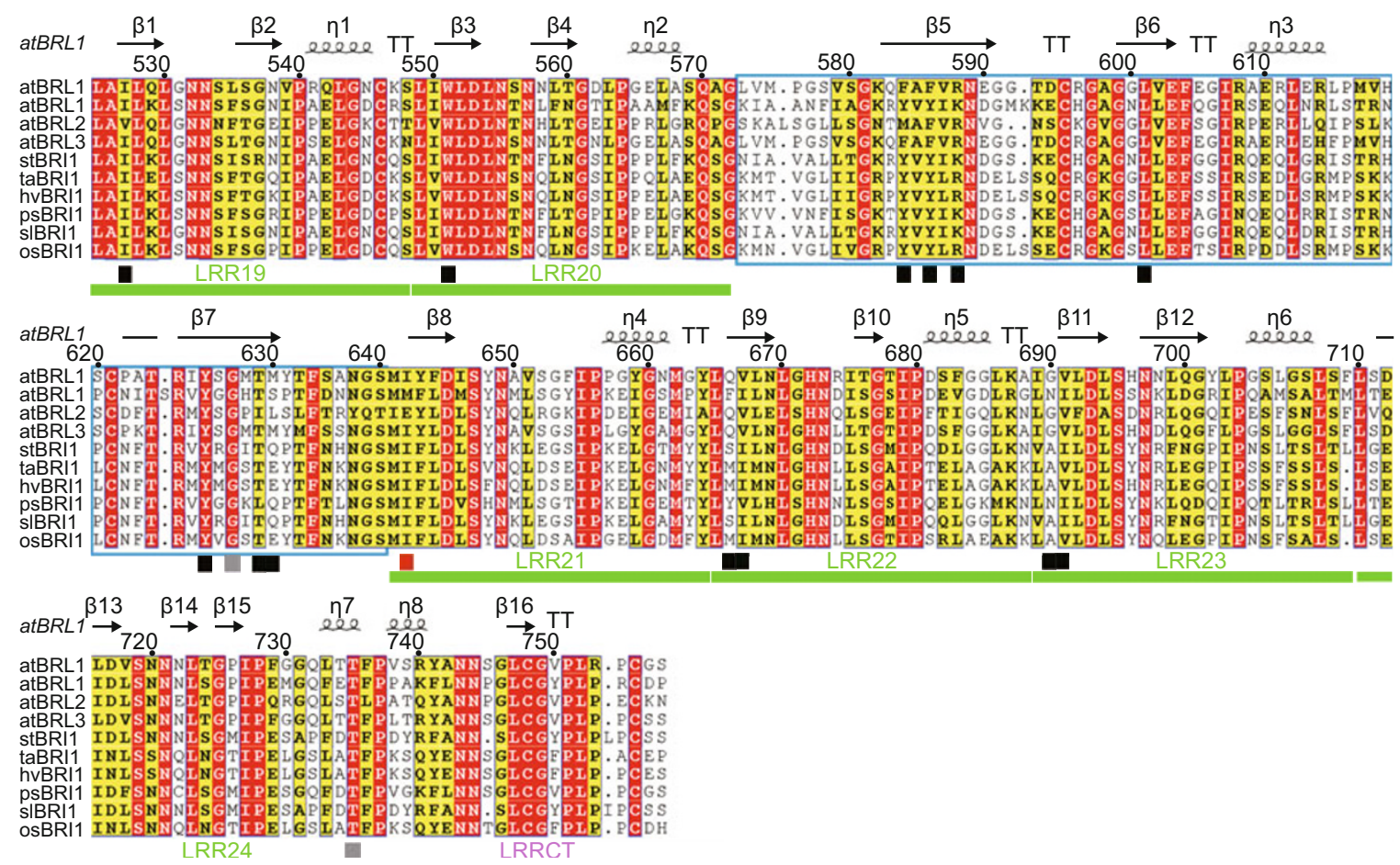

Figure 4. Sequence alignment of brassinolide binding pockets of BRI1 and its homologs. Conserved and similar residues are highlighted with red and yellow grounds respectively. The island domain is highlighted within the blue square. Residues involved in brassinolide recognition of BRL1 are indicated with black solid squares at bottom. Red and gray solid squares indicate residues corresponding to Glu614 of BRL2, Gly644 and Thr750 of BRI1. "LRRCT" represents LRR C-terminal cap. "at", "st", "ta", "hv", "ps", "sl" and "os" represent Arabidopsis thaliana, Solanum tuberosum, Triticum aestivum, Hordeum vulgare, Pisum sativumand, Solanum lycopersicum, Oryza sativa, respectively.

land domain are conserved between BRL1 and BRI1, the nonconserved Arg588 of BRL1 makes tight packing against the carbonyl oxygen in ring $\mathrm{B}$ of brassinolide via cation-pi interaction (Fig. 3B and 3C), further blocking brassinolide from solvent region. In contrast, Lys601 in BRI1, the equivalent of Arg588 in BRL1, is not directly involved in interaction with brassinolide. The varied residues of the brassinolide-binding pocket from the LRR domain appear to be critical for a deeper binding of brassinolide to BRL1(LRR). In the structure of brassinolidebound BRI1(LRR), Asn705 supports brassinolide via contact of its hydrophobic portion with ring A (Fig. $3 \mathrm{C}$ ), thus blocking brassinolide from binding further to the bottom of the binding pocket. In BRL1, Gly690 with no side chain substitutes Asn705 of BRI1, allowing ring A of brassinolide to fall down and form Van Der Waals interactions with Val667, Gly690 and Val691 from underneath (Fig. 3C). Together, our structural analyses offer an explanation for the higher affinity of brassinolide with BRL1 than with BRI1.

\section{Structural model of BRL2}

$B R L 2$, unlike BRL1 or BRL3, is unable to recover the wild type phenotype of a bri1 mutant and has no specific binding to brassinolide despite their high sequence homology (CanoDelgado et al., 2004). These results appear a little surprising, because the residues around the brassinolide binding site are largely conserved among the three proteins (Fig. 4). In fact, BRL2 (residues 497-729) has a higher sequence homology with BRL1 than with BRI1. Additionally, the two residues, Arg588 and Gly690 of BRL1 that contribute to a higher binding affinity to brassinolide are also conserved in BRL2 (Fig. 4). These data suggest that there exist other non-conserved residues in BRL2 responsible for its inability to recognize brassinolide. The high sequence homology with BRL1 allowed us to build a structure model of BRL2 with high confidence. We therefore modeled a structure for BRL2 (residues 497-729) using BRL1 as the template with the program MODELLER (Eswar et al., 2008). The modeled partial BRL2 structure shows that the bulky and negatively-charged residue Glu614 substitutes lle642 of BRL1, which is located at the inner end of brassinolide binding pocket (Fig. 5). We explored all possible rotamers of the side chain of Glu614 in COOT and found that one preferred rotamer without steric clashes with other parts of the binding pocket is close to the hydrophobic cavity anchoring the side chain of brassinolide (Fig. 5B). Thus, this mutation in BRL2 may act to disrupt the brassinolide binding by changing the hydrophobicity of this area. Supporting the significance of this hydrophobic cavity in brassinolide recognition by BRI1, introduction of polar groups at the end of side chain significantly attenuates brassinolide bioactivity (Back and Pharis, 2003) 

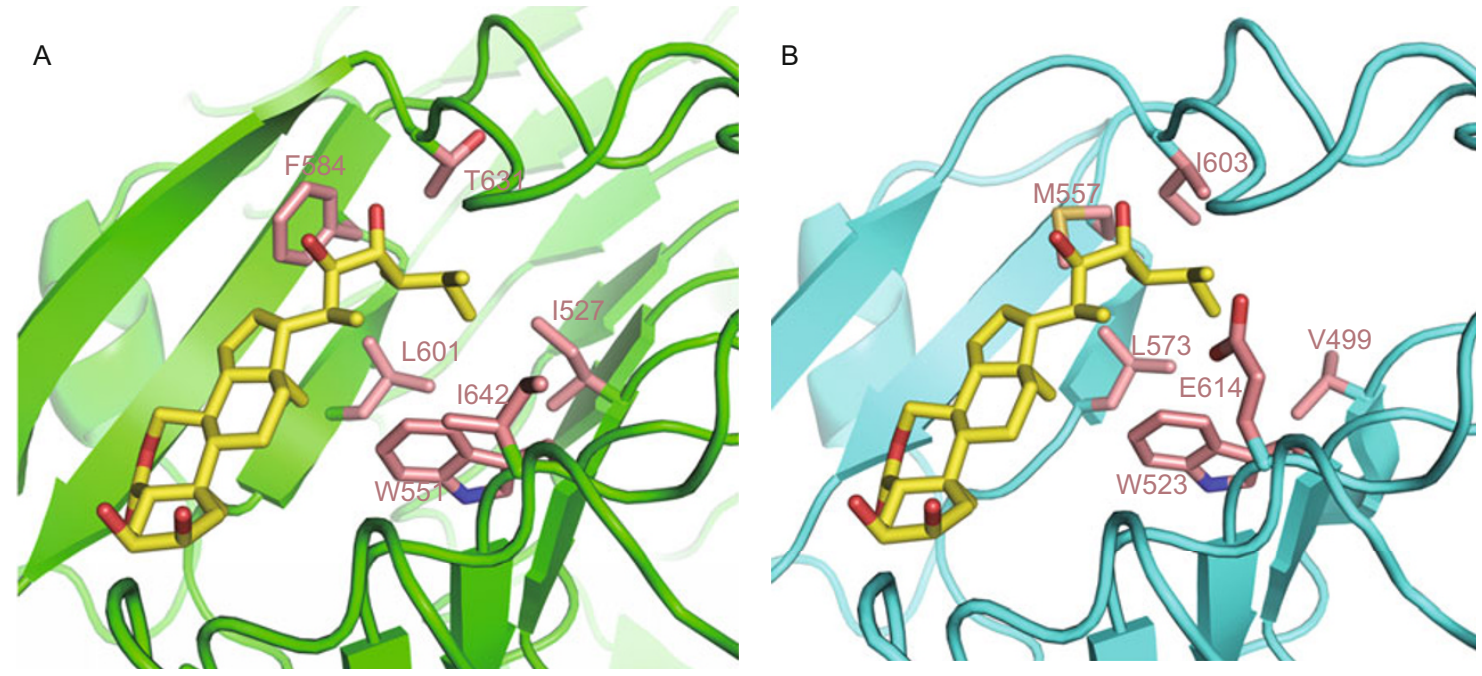

Figure 5. Comparation of the brassinolide binding pockets of BRL1 and BRL2. The brassinolide binding pockets of BRL1 and the modeled BRL2 structure are shown in green and cyan cartoons, respectively. Colored in salmon are the residues close to the side chain of brassinolide. Brassinolide is depicted as yellow stick.

and the hydroxylation of C26 by an enzyme BAS1 is shown to be one way to inactivate brassinosteroids in plants (Neff et al., 1999).

\section{DISCUSSION}

We have solved the crystal structure of the BRI1 homolog BRL1 in complex with brassinolide in the current study. While BRI1(LRR) and BRL1(LRR) share a highly conserved structure (Fig. 3A and 4), the subtle differences around their brassinolide binding sites generate striking effects on their ability to recognize brassinolide (Cano-Delgado et al., 2004) (Fig. 3C). The interaction with Arg588 of BRL1 contributes to a higher binding affinity of brassinolide with BRL1 than with BRI1 (Fig. 3C). The non-conserved Gly690 of BRL1 appears to further strengthen its recognition of brassinolide (Figs. $3 \mathrm{C}$ and 4). The fact that many BRI1 homologs harbor a small side chain residue (Ala or Gly) at the corresponding position of Gly690 of BRL1 suggests a conserved role played by this residue in recognizing brassinolide (Fig. 4). Our modeling study suggests that a change in one residue around the side chain binding cavity can have a deleterious effect on the recognition of brassinolide by BRI1 family of receptors (Fig. 5). Interestingly, this residue, Glu614 of Arabidopsis BRL2, is conserved in rice BRL2 (OsBRL2) (Nakamura et al., 2006), but not in other BRI1 homologs (Fig. 4), suggesting that it may be specific for BRL2 function. However, it is unclear why BRL3 exhibited a low affinity with brassinolide than BRL1, because all amino acids involved in brassinolide recognition are conserved between BRL1 and BRL3 (Fig. 4). The lack of commercially available radio-labeled brassinolide as well as the extremely low solubility of brassinolide rendered it difficult for us to test the non-conserved residues critical for selection of BRI1 family of receptors by brassinolide. Nonethe- less, our data provide some structural insights for differential binding of brassinolide to the BRI1 family of receptors and allow a more efficient design of plant growth regulators for agricultural practice.

The mechanism of receptor activation of BRI1 family receptors is not fully clear. The crystal packing and the gel filtration (Hothorn et al., 2011; She et al., 2011) assay didn't support the model in which ligand induces dimerization of the extracelluar domains of BRI1. The most marked conformational change upon hormone binding occurs to the interdomain loops that connect the island domain and LRRs in the structure of BRI1(LRR) (She et al., 2011). In BRL1(LRR), the interdomain loops also adopt similar conformations with BRI1(LRR) (Fig. 3A). This conformation change may thus create a binding surface to interact with other protein. Two BRI1 mutants Gly644Asp and Thr750lle, located around the ligand binding pocket (Fig. 3A), show loss-of-function phenotypes (Noguchi et al., 1999; Friedrichsen et al., 2000) but still bind brassinolide well (Wang et al., 2001; Kinoshita et al., 2005). These two residues are conserved among the BRI1 family receptors (Fig. 4), which may be key residues in extracelluar domain responsible for receptor activation. When animals' steroid hormones are recognized by nuclear receptors, they are almost fully buried into the largely helical structures of ligand binding domains of their receptors and their binding can induce a dramatic shift of the C-termial activation helix to initiate downstream signaling (Renaud and Moras, 2000; Huang et al., 2010). In contrast, one side of the fused ring of brassinosteroid is nearly solvent exposed in the structures of BRI1(LRR) and BRL1(LRR) (Fig. 3A). This unshielded region may provide additional sites for protein-protein interaction. The extracelluar domain of the BRI1 coreceptor, BAK1, has been shown to provide a platform for receptor/coreceptor complex formation (Jaillais et al., 
2011a). Therefore, it is likely that the extracelluar domains of BAK1 and BRI1 form heterodimers to trigger the downstream signaling pathway. However, it still cannot exclude the possibility that some unknown players may bridge the interaction of extracelluar domains of BAK1 and BRI1 family proteins, as suggested by others (Nam and Li, 2002). Further structural and functional studies are required to elucidate the mechanism for the activation of BRI1 family receptors.

\section{MATERIALS AND METHODS}

Protein expression and purification

The LRR domain of BRL1 (residues 25-758) from Arabidopsis with an engineered C-terminal $6 \times$ His tag was generated by standard PCRbased cloning strategy and its identity was confirmed by sequencing. The protein was expressed in sf21 cells using the vector pFastBac 1 (Invitrogen) with a modified $\mathrm{N}$-terminal Hemolin peptide. One litre of cells $\left(2.0 \times 10^{6}\right.$ cells $\left./ \mathrm{mL}\right)$ was infected with $20 \mathrm{~mL}$ baculovirus using a multiplicity of infection of 4 at $28^{\circ} \mathrm{C}$, and protein was harvested from the media after $48 \mathrm{~h}$. The protein was purified using Ni-NTA (Novagen) and size-exclusion chromatography (Superdex 200, Pharmacia) in buffer $(10 \mathrm{mmol} / \mathrm{L}$ Tris, $\mathrm{pH} 8.0,100 \mathrm{mmol} / \mathrm{L} \mathrm{NaCl})$ at $4^{\circ} \mathrm{C}$. For crystallization of $B R L 1(L R R)$, the purified protein was concentrated to about $3.0 \mathrm{mg} / \mathrm{mL}$.

Crystallization, data collection, structure determination and refinement

Crystals of BRL1(LRR) were generated by mixing the protein with an equal amount of well solution $(1 \mathrm{~mL})$ by the hanging-drop vapordiffusion method. A mixture of brassinolide (in DMSO) and BRL1(LRR) with a molar ratio of $10: 1$ was centrifuged at $14,000 \mathrm{~g}$ for $2 \mathrm{~min}$ to remove insoluble brassinolide and then used for generating crystals of their complex. The buffer producing crystals of brassinolide-bound BRL1(LRR) contained $0.2 \mathrm{~mol} / \mathrm{L}$ ammonium citrate dibasic and $20 \%$ $(w / v)$ polyethylene glycol (PEG) 3350 . Crystals grew to their maximum size $\left(0.1 \times 0.1 \times 0.05 \mathrm{~mm}^{3}\right)$ within 7 days at $18^{\circ} \mathrm{C}$. The diffraction data set was collected at the Shanghai Synchrotron Radiation Facility (SSRF) at beam line BL17U1 using a CCD detector. Crystals of brassinolide-bound BRL1(LRR) belong to space group C2. For data collection, the crystals were equilibrated in a cryoprotectant buffer containing reservoir buffer plus $20.0 \%(v / V)$ glycerol. The data were processed using HKL2000 (Otwinowski and Minor, 1997). Molecular replacement (MR) with the program PHASER (McCoy et al., 2007) was used to solve the crystal structure of brassinolide-bound BRL1(LRR). The atomic coordinates of BRI1 (PDB ID: 3RGZ) were used as the initial searching model. The model from MR was built with the program COOT (Emsley and Cowtan, 2004) and subsequently subjected to refinement by the program PHENIX (Adams et al., 2002). The structure figures were prepared using PyMOL (http://www.pymol.org).

\section{Homology modeling of BRL2}

Homology model of BRL2 (residues 497-729) was built with MODELLER (Eswar et al., 2008) using BRL1 as the template. BRL2 (residues $497-729$ ) shares $\sim 53 \%$ sequence identity with BRL1.

\section{ACCESSION CODE}

Protein Data Bank: The atomic coordinates and structure factors of brassinolide-bound BRL1(LRR) have been deposited with accession code 4JOM.

\section{ACKNOWLEDGMENTS}

We thank F. Yu and J. He at Shanghai Synchrotron Radiation Facility (SSRF) for assistance with data collection. This work was funded by the State Key Program of National Natural Science of China (No. 31130063) and the National Basic Research Program (973 Program) (No. 2010CB835300).

\section{ABBREVIATIONS}

$B R L, B R I$ 1-like receptor kinase; BRI1, brassinosteroid-insensitive 1; $B R L 1(L R R)$, LRR domain of BRL1; LRR, leucine-rich repeat; LRR$R L K$, leucine-rich repeat receptor like kinase

\section{COMPLIANCE WITH ETHICS GUIDELINES}

Ji She, Zhifu Han, Bin Zhou, and Jijie Chai declare that they have no conflict of interest.

This article does not contain any studies with human or animal subjects performed by the any of the authors.

\section{REFERENCES}

Adams, P.D., Grosse-Kunstleve, R.W., Hung, L.W., loerger, T.R., McCoy, A.J., Moriarty, N.W., Read, R.J., Sacchettini, J.C., Sauter, N.K., and Terwilliger, T.C. (2002). PHENIX: building new software for automated crystallographic structure determination. Acta Crystallogr D Biol Crystallogr 58, 1948-1954.

Back, T.G., and Pharis, R.P. (2003). Structure-activity studies of brassinosteroids and the search for novel analogues and mimetics with improved bioactivity. J Plant Growth Regul 22, 350-361.

Cano-Delgado, A., Yin, Y., Yu, C., Vafeados, D., Mora-Garcia, S., Cheng, J.C., Nam, K.H., Li, J., and Chory, J. (2004). BRL1 and BRL3 are novel brassinosteroid receptors that function in vascular differentiation in Arabidopsis. Development 131, 5341-5351.

Ceserani, T., Trofka, A., Gandotra, N., and Nelson, T. (2009). VH1/BRL2 receptor-like kinase interacts with vascular-specific adaptor proteins VIT and VIK to influence leaf venation. Plant J 57, 1000-1014.

Clay, N.K., and Nelson, T. (2002). VH1, a provascular cell-specific receptor kinase that influences leaf cell patterns in Arabidopsis. Plant Cell 14, 2707-2722.

Clouse, S.D., and Sasse, J.M. (1998). BRASSINOSTEROIDS: Essential Regulators of Plant Growth and Development. Annu Rev Plant Physiol Plant Mol Biol 49, 427-451.

Di Matteo, A., Federici, L., Mattei, B., Salvi, G., Johnson, K.A., Savino, C., De Lorenzo, G., Tsernoglou, D., and Cervone, F. (2003). The crystal structure of polygalacturonase-inhibiting protein (PGIP), a leucine-rich repeat protein involved in plant defense. Proc Natl Acad Sci U S A 100, 10124-10128.

Emsley, P., and Cowtan, K. (2004). Coot: model-building tools for molecular graphics. Acta Crystallogr D Biol Crystallogr 60, 2126-2132. 
Eswar, N., Eramian, D., Webb, B., Shen, M.Y., and Sali, A. (2008). Protein structure modeling with MODELLER. Methods Mol Biol 426, 145-159.

Friedrichsen, D.M., Joazeiro, C.A., Li, J., Hunter, T., and Chory, J. (2000). Brassinosteroid-insensitive-1 is a ubiquitously expressed leucine-rich repeat receptor serine/threonine kinase. Plant Physiol 123, 1247-1256.

Fukuda, H. (2004). Signals that control plant vascular cell differentiation. Nat Rev Mol Cell Biol 5, 379-391.

He, Z., Wang, Z.Y., Li, J., Zhu, Q., Lamb, C., Ronald, P., and Chory, J. (2000). Perception of brassinosteroids by the extracellular domain of the receptor kinase BRI1. Science 288, 2360-2363.

Hothorn, M., Belkhadir, Y., Dreux, M., Dabi, T., Noel, J.P., Wilson, I.A., and Chory, J. (2011). Structural basis of steroid hormone perception by the receptor kinase BRI1. Nature 474, 467-471.

Huang, P., Chandra, V., and Rastinejad, F. (2010). Structural overview of the nuclear receptor superfamily: insights into physiology and therapeutics. Ann Rev Physiol 72, 247-272.

Jaillais, Y., Belkhadir, Y., Balsemao-Pires, E., Dangl, J.L., and Chory, J. (2011a). Extracellular leucine-rich repeats as a platform for receptor/coreceptor complex formation. Proc Natl Acad Sci U S A 108, 8503-8507.

Jaillais, Y., Hothorn, M., Belkhadir, Y., Dabi, T., Nimchuk, Z.L., Meyerowitz, E.M., and Chory, J. (2011b). Tyrosine phosphorylation controls brassinosteroid receptor activation by triggering membrane release of its kinase inhibitor. Genes Dev 25, 232-237.

Kim, T.W., and Wang, Z.Y. (2010). Brassinosteroid signal transduction from receptor kinases to transcription factors. Annu Rev Plant Biol 61, 681-704.

Kinoshita, T., Cano-Delgado, A., Seto, H., Hiranuma, S., Fujioka, S., Yoshida, S., and Chory, J. (2005). Binding of brassinosteroids to the extracellular domain of plant receptor kinase BRI1. Nature 433, 167-171.

Li, J., and Chory, J. (1997). A putative leucine-rich repeat receptor kinase involved in brassinosteroid signal transduction. Cell 90 , 929-938.

Li, J., Wen, J., Lease, K.A., Doke, J.T., Tax, F.E., and Walker, J.C. (2002). BAK1, an Arabidopsis LRR receptor-like protein kinase, interacts with BRI1 and modulates brassinosteroid signaling. Cell 110, 213-222.

McCoy, A.J., Grosse-Kunstleve, R.W., Adams, P.D., Winn, M.D., Storoni, L.C., and Read, R.J. (2007). Phaser crystallographic software. J Appl Crystallogr 40, 658-674.

Nakamura, A., Fujioka, S., Sunohara, H., Kamiya, N., Hong, Z., Inukai,
Y., Miura, K., Takatsuto, S., Yoshida, S., Ueguchi-Tanaka, M., et al. (2006). The role of OsBRI1 and its homologous genes, OsBRL1 and OsBRL3, in rice. Plant Physiol 140, 580-590.

Nam, K.H., and Li, J. (2002). BRI1/BAK1, a receptor kinase pair mediating brassinosteroid signaling. Cell 110, 203-212.

Neff, M.M., Nguyen, S.M., Malancharuvil, E.J., Fujioka, S., Noguchi, T., Seto, H., Tsubuki, M., Honda, T., Takatsuto, S., Yoshida, S., et al. (1999). BAS1: A gene regulating brassinosteroid levels and light responsiveness in Arabidopsis. Proc Natl Acad Sci U S A 96, 15316-15323.

Noguchi, T., Fujioka, S., Choe, S., Takatsuto, S., Yoshida, S., Yuan, H., Feldmann, K.A., and Tax, F.E. (1999). Brassinosteroid-insensitive dwarf mutants of Arabidopsis accumulate brassinosteroids. Plant Physiol 121, 743-752.

Otwinowski, Z., and Minor, W. (1997). Processing of X-ray diffraction data collected in oscillation mode. Methods Enzymol 276, 307-326.

Renaud, J.P., and Moras, D. (2000). Structural studies on nuclear receptors. Cell Mol Life Sci 57, 1748-1769.

She, J., Han, Z., Kim, T.W., Wang, J., Cheng, W., Chang, J., Shi, S., Yang, M., Wang, Z.Y., and Chai, J. (2011). Structural insight into brassinosteroid perception by BRI1. Nature 474, 472-476.

Shiu, S.H., and Bleecker, A.B. (2001). Receptor-like kinases from Arabidopsis form a monophyletic gene family related to animal receptor kinases. Proc Natl Acad Sci U S A 98, 10763-10768.

Vert, G., Nemhauser, J.L., Geldner, N., Hong, F., and Chory, J. (2005). Molecular mechanisms of steroid hormone signaling in plants. Annu Rev Cell Dev Biol 21, 177-201.

Wang, X., and Chory, J. (2006). Brassinosteroids regulate dissociation of $\mathrm{BKI} 1$, a negative regulator of BRI1 signaling, from the plasma membrane. Science 313, 1118-1122.

Wang, X., Kota, U., He, K., Blackburn, K., Li, J., Goshe, M.B., Huber, S.C., and Clouse, S.D. (2008). Sequential transphosphorylation of the BRI1/BAK1 receptor kinase complex impacts early events in brassinosteroid signaling. Dev Cell 15, 220-235.

Wang, Z.Y., Bai, M.Y., Oh, E., and Zhu, J.Y. (2012). Brassinosteroid signaling network and regulation of photomorphogenesis. Annu Rev Genet 46, 701-724.

Wang, Z.Y., Seto, H., Fujioka, S., Yoshida, S., and Chory, J. (2001). BRI1 is a critical component of a plasma-membrane receptor for plant steroids. Nature 410, 380-383.

Zhou, A., Wang, H., Walker, J.C., and Li, J. (2004). BRL1, a leucinerich repeat receptor-like protein kinase, is functionally redundant with BRI1 in regulating Arabidopsis brassinosteroid signaling. Plant J 40, 399-409. 